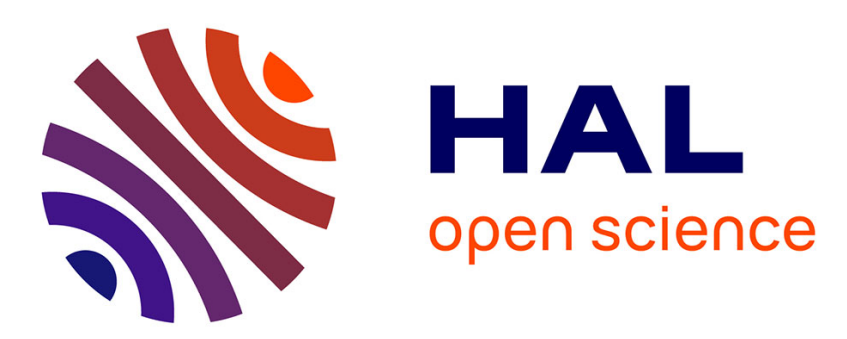

\title{
OMNIDIRECTIONAL IMAGE PROCESSING USING GEODESIC METRIC
}

Cédric Demonceaux, Pascal Vasseur

\section{To cite this version:}

Cédric Demonceaux, Pascal Vasseur. OMNIDIRECTIONAL IMAGE PROCESSING USING GEODESIC METRIC. IEEE International Conference on Image Processing - ICIP, Nov 2009, Cairo, Egypt. hal-01779777

\section{HAL Id: hal-01779777 https://hal.science/hal-01779777}

Submitted on 26 Apr 2018

HAL is a multi-disciplinary open access archive for the deposit and dissemination of scientific research documents, whether they are published or not. The documents may come from teaching and research institutions in France or abroad, or from public or private research centers.
L'archive ouverte pluridisciplinaire HAL, est destinée au dépôt et à la diffusion de documents scientifiques de niveau recherche, publiés ou non, émanant des établissements d'enseignement et de recherche français ou étrangers, des laboratoires publics ou privés. 


\title{
OMNIDIRECTIONAL IMAGE PROCESSING USING GEODESIC METRIC
}

\author{
C. Demonceaux and P. Vasseur
}

\author{
M.I.S. EA 4290, \\ 33 rue Saint Leu \\ 80039 Amiens Cedex 1, France \\ cedric.demonceaux@u-picardie.fr pascal.vasseur@u-picardie.fr
}

\begin{abstract}
Due to distorsions of catadioptric sensors, omnidirectional images can not be treated as classical images. If the equivalence between central catadioptric images and spherical images is now well known and used, spherical analysis often leads to complex methods particularly tricky to employ. In this paper, we propose to derive omnidirectional image treatments by using geodesic metric. We demonstrate that this approach allows to adapt efficiently classical image processing to omnidirectional images.
\end{abstract}

Index Terms- Omnidirectional image, image processing

\section{INTRODUCTION}

Numerous applications benefit from the use of cameras with panoramic field of view [1]. However, because of distorsions observed in images obtained with this kind of sensor (fig 3) classical image processing treatments are not adapted.

Consequently, Bogdanova and al. [2] proposed to include the deformation of the mirror by considering the Jacobian induced by the mirror geometry. This method permits the correction of image distorsions for gradient estimation but can not be extended to image filtering.

An other approach consists in considering omnidirectional image as a planar projection of a spherical image. Indeed, [3] [4], [5] have demonstrated that a central image (catadioptric or perspective) or a fisheye image are equivalent to a spherical image if the intrinsic parameters of the sensor are known. In this way, spherical space appears more suitable for image processing than Euclidean plane. In order to avoid spherical geometry, Jacquey and al. [6] proposed to project the spherical image onto an including virtual cylinder. Classical image filterings are then applied on this cylinder. Even if this representation appears to be a good approximation, cylindric geometry is not adapted to spherical images. Demonceaux and Vasseur [7] proposed to adapt Markovian methods to omnidirectional images by defining a neighborhood with spherical constraints for each pixel. This representation presents a real improvement of the quality of the results obtained with

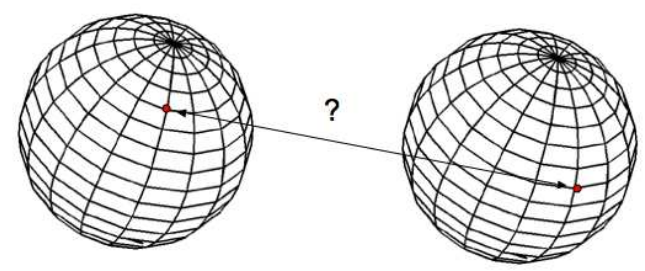

Fig. 1. Sphere sampling with constant $\phi$ and $\theta$ provides an irregular grid.

Markovian treatments but can not be also generalized to image filtering.

In [8] and [9], omnidirectional are considered as spherical images and the authors use spherical harmonics in order to define convolution product and Fourier transform. However, these spherical tools have been developped from a sampling which is not appropriate to omnidirectional images. Indeed, spherical coordinates of a point are defined as

$$
\forall x \in \mathcal{S}^{2}, x=(\cos (\phi) \sin (\theta), \sin (\phi) \sin (\theta), \cos (\theta)),
$$

where $\phi \in[0,2 \pi[, \theta \in[0, \pi]$ and the natural sampling of the sphere consists in considering every points separated by constant $\theta_{i}$ and $\phi_{j}$. Thus, the distance of two points on this kind of grid depends on their latitude (fig 1). Consequently, this irregular grid requires to introduce a weighting on the azimuth derivate for computing the gradient $\frac{\partial I}{\partial \phi}([9])$ :

$$
\nabla_{\mathcal{S}^{2}} I(\theta, \phi)=\frac{\partial I}{\partial \theta} e_{\theta}(\theta, \phi)+\frac{1}{\sin (\theta)} \frac{\partial I}{\partial \phi} e_{\phi}(\theta, \phi)
$$

Moreover, this representation leads to a non-commutative convolution product and does not verify the useful following relation $(I * f)^{\prime}=I * f^{\prime}$. In this way, computing the gradient by using the low-pass filter derivative as in perspective images is impossible.

Ainouz and al [10] propose to filter an omnidirectional image using the projection of the previous spherical sampling on the omnidirectional plane. But, once more, due to this irregular sampling, the formulation is not really correct. 
In this paper, we propose to define omnidirectional image convolution from geodesic distance between points. We demonstrate that this definition allows to use similar filters than in perspective image processing and moreover, that commutative properties and derivative computation of the convolution remain valid.

\section{GEODESIC METRIC}

Perspective image processing tools are generally based on Euclidean metric :

$$
\forall x, y \in \mathbb{R}^{2}, d(x, y)=\|x-y\|_{l^{2}\left(\mathbb{R}^{2}\right)} .
$$

From this metric, it is possible to define a neighborhood in order to compute derivatives, to detect corners or to perform point matching for instance. The aim of the neighborhood is to define in the image plane the dependency between 3D points. Practically, for an orthographic camera this neighborhood describes exactly 3D dependency of points if they live on a fronto-parallel plane (fig 2(a)). For a perspective camera, this neighborhood is an approximation whatever the configuration of 3D points (fig 2(b)). In the case of an omnidirectional image, this neighborhood is no more adapted (fig 2(c)). However, if we consider a regular sampling according to $\theta$ and $\phi$ (fig 2(d)), this neighborhood describes exactly the mutual influence of 3D points if they live on a concentric sphere of the unitary sphere and is a good approximation in the general case. This is this kind of neighborhood that we develop in the following.

\subsection{Definition}

Let $\mathrm{P}$, the projection of an omnidirectional planar image into its equivalent spherical image:

$$
\mathrm{P}: \begin{array}{ccc}
\mathbb{R}^{2} & \rightarrow & S^{2} \\
x & \mapsto & x_{s}=(\theta, \phi)
\end{array}
$$

In order to apply image processing treatments on the sphere, we propose to employ the following geodesic distance :

$$
\forall x_{s}, y_{s} \in \mathcal{S}^{2} \quad d\left(x_{s}, y_{s}\right)=\operatorname{arcos}\left(x_{s}, y_{s}\right) .
$$

Let $x_{s} \in \mathcal{S}^{2}$, the projection of planar image pixel $x$ on the equivalent sphere $\left(x_{s}=\mathrm{P}(x)\right)$. The continuous neighborhood $\mathcal{V}_{r}(x)$ of pixel $x$ in the image is defined as :

$$
\mathcal{V}_{r}(x)=\left\{y_{s} \in \mathcal{S}^{2}, d\left(x_{s}, y_{s}\right)<r\right\}
$$

The discrete neighborhood $\mathcal{V}_{r}^{N}(x)$ is then defined by (fig. 2(d)):

$$
\begin{aligned}
& \forall \quad \mathrm{P}(x)=x_{s}=(\theta, \phi) \in \mathcal{S}^{2} \\
& \mathcal{V}_{r}^{N}(x)=\left\{\left(\theta^{\prime}, \phi^{\prime}\right) \mid\left\{\begin{array}{l}
\theta^{\prime}=\theta+n r, \\
\phi^{\prime}=\phi+\frac{p}{\sin (\theta)} r
\end{array} \quad-N \leq n, p \leq N\right\}\right.
\end{aligned}
$$

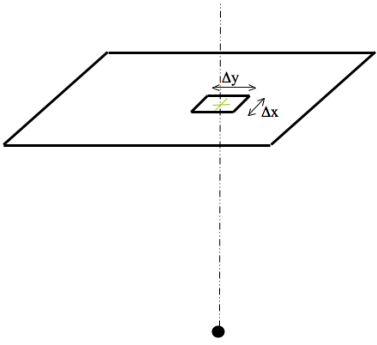

(a)

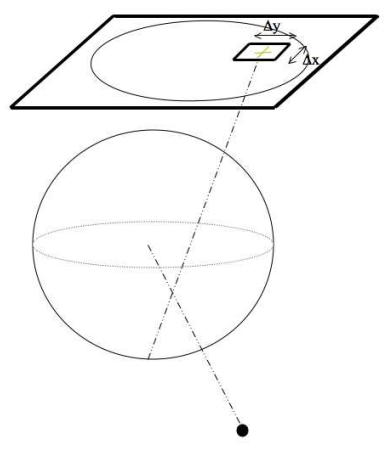

(c)

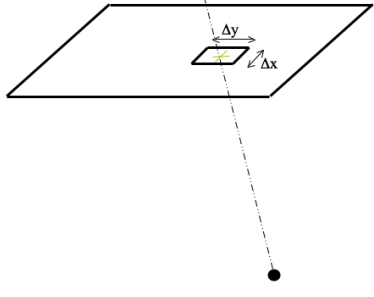

(b)

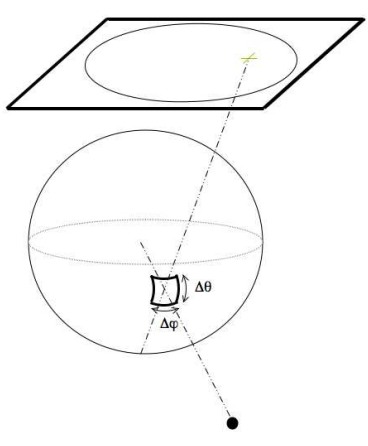

(d)
Fig. 2. Image formation and neighborhood dependency. (a) Orthographic image, Euclidean metric, (b) perspective image, Euclidean metric, (c) omnidirectional image, Euclidean metric, (d) omnidirectional image, geodesic metric.

By analogy with the planar case, we define a regular grid of $(2 N+1) \times(2 N+1)$ points centered in $x_{s}$ such as the geodesic distance between a point and its nearest neighbours is exactly equal to $r$ :

$$
\forall y_{s} \in \mathcal{V}_{r}^{N}(x) \quad \min _{z_{s} \in \mathcal{V}_{r}^{N}(x)} d\left(y_{s}, z_{s}\right)=r
$$

\subsection{Filtering}

Considering an omnidirectional image $I$ and $I_{\mathcal{V}_{r}^{N}(x)}=$ $I\left(\mathrm{P}^{-1}\left(\mathcal{V}_{r}^{N}(x)\right)\right)$ as a regular grid centered in $x$ with $(2 N+$ $1)^{2}$ pixels associated to their grey level values.

Let $H$ be a filter of size $(2 N+1) \times(2 N+1)$, the filtered image $I H$ at the point $x=\left(x_{1}, x_{2}\right)$ can be defined similarly than in the classical case by the convolution product ${ }^{1}$ :

$$
\begin{aligned}
I H(x) & =I_{\mathcal{V}_{r}^{N}(x)}(x) * H \\
& =\sum_{i=-N}^{N} \sum_{j=-N}^{N} I_{\mathcal{V}_{r}^{N}(x)}\left(x_{1}-i, x_{2}-j\right) H(i, j)
\end{aligned}
$$

\footnotetext{
${ }^{1}$ This representation is equivalent to the convolution product on the tangent plane at point $x_{s}=\mathrm{P}(x)$.
} 
If we consider the particular example of the gradient from a Sobel filter,

$$
S=\left(\begin{array}{ccc}
-1 & -2 & -1 \\
0 & 0 & 0 \\
1 & 2 & 1
\end{array}\right)
$$

we then obtain :

$$
\|\left.\nabla I(x)\right|^{2}=\left|I_{\mathcal{V}_{r}^{2}(x)} * S\right|^{2}+\left|I_{\mathcal{V}_{r}^{2}(x)} * S^{\prime}\right|^{2}
$$

In the same way, image filtering with a Gaussian can be seen as a weighting of the points according to their distance from the considered point. Consequently, the traditional Gaussian defined as :

$$
G_{x}(y, \sigma)=\frac{1}{2 \pi \sigma^{2}} \exp -\frac{\|x-y\|^{2}}{2 \sigma^{2}}
$$

can be replaced by :

$$
G_{x}(y, \sigma)=\frac{1}{2 \pi \sigma^{2}} \exp -\frac{d(x, y)}{2 \sigma^{2}}
$$

Moreover, it is possible to develop the following Laplacian of Gaussian :

$$
L o G_{x}(y)=-\frac{1}{\pi \sigma^{4}}\left[1-\frac{d(x, y)}{2 \sigma^{2}}\right] \exp -\frac{d(x, y)}{2 \sigma^{2}}
$$

Thus, while harmonic analysis proposes definitions for Gaussian and Laplacian of Gaussian very tricky to implement for spherical image processing [9], our approach allows to adapt easily classical filters. Indeed, thanks to $(4,5)$ and the convolution product defined by (3), the masks used in perspective image processing remain valid.

\section{EXPERIMENTS}

In this section, we demonstrate the benefits of our modeling in the case of corner detection based on Harris method and in the case of point matching based on ZNCC. It is worth noting that our geodesic neighborhood requires a first interpolation phase. In the following experiments of this section, we obtain $I_{\mathcal{V}_{r}^{N}(x)}$ by a nearest neighbour interpolation.

\subsection{Harris detector}

Harris detector allows to extract corners in images and is particularly useful in computer vision. In perspective image processing, detect corners by Harris method consists in studying eigenvalues of matrix $M$ for each point $X$ :

$$
M(X)=\left[\begin{array}{cc}
L_{x}^{2}(X, \sigma) & L_{x} L_{y}(X, \sigma) \\
L_{x} L_{y}(X, \sigma) & L_{y}^{2}(X, \sigma)
\end{array}\right]
$$

where $L_{i}(X, \sigma)=\frac{\partial}{\partial i} G_{X}(X, \sigma) * I(X)$

In the case of omnidirectional images, this definition is always valid by using the convolution defined in (3) and (4). Results
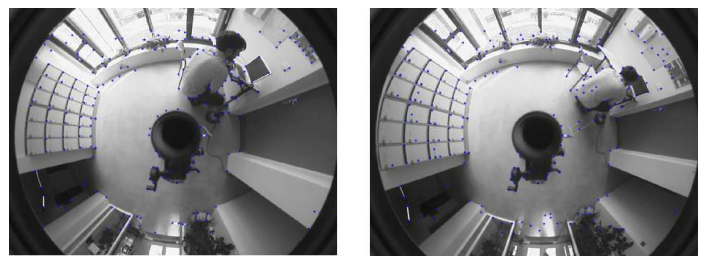

(a)
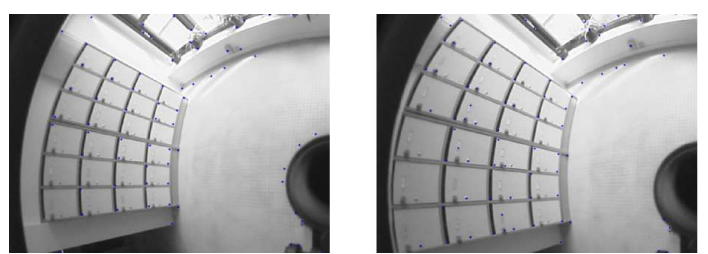

(b)
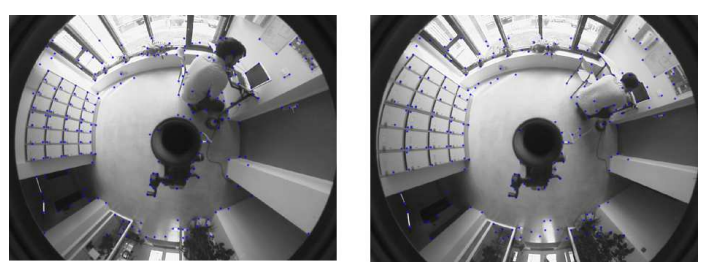

(c)
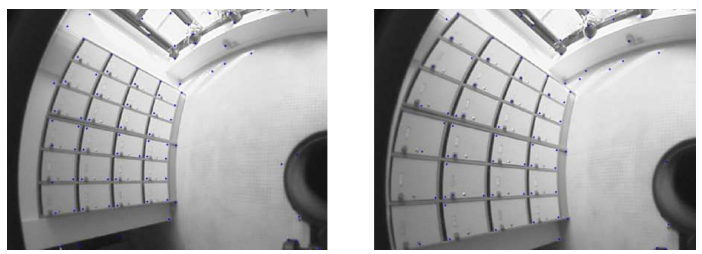

(d)

Fig. 3. Harris detector. (a)-(b) classical approach,(c)-(d) geodesic approach

of Harris detector are shown in figure 3 for two consecutive images of a sequence. Table 1 presents the repeatability factor [11] of our detector in comparison with the classical Harris detector. On the locker (fig 3(b) and 3(d)), 50 corners have been detected in $I_{1}$ with our method and 55 with the classical approach. In the second frame, we detect 51 corners with our approach while the classical Harris detector finds only 36 corners. In the results of the classical Harris detector, only 29 common corners have been detected which corresponds to a repeatability rate equal to $80 \%$. In the case of the geodesic approach, 43 common corners have been detected between the two images which corresponds to a repeatability of $86 \%$.

\subsection{Matching with $\mathrm{ZNCC}$}

Beyond image processing methods based on filtering, point matching techniques based on correlation have also to include 


\begin{tabular}{|c|r|l|r|l|}
\hline & \multicolumn{2}{|c|}{ Geodesic Harris } & \multicolumn{2}{c|}{ Classical Harris } \\
\hline Image & $I_{1}$ & $I_{2}$ & $I_{1}$ & $I_{2}$ \\
\hline Corners & 231 & 227 & 212 & 239 \\
\hline Corners on locker & 50 & 51 & 55 & 36 \\
\hline Repeatability & \multicolumn{2}{|c|}{$43 \rightarrow 86 \%$} & \multicolumn{2}{|c|}{$29 \rightarrow 80 \%$} \\
\hline
\end{tabular}

Table 1.

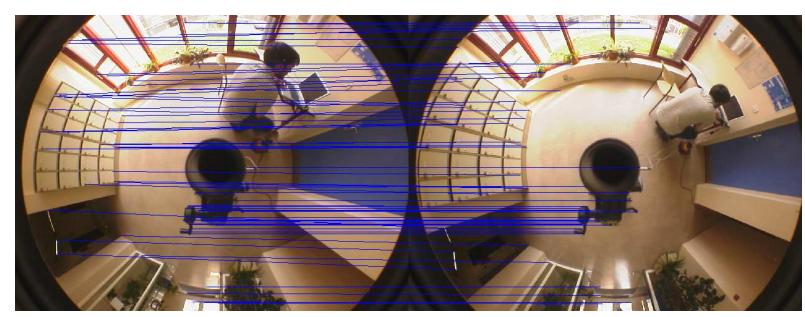

(a)

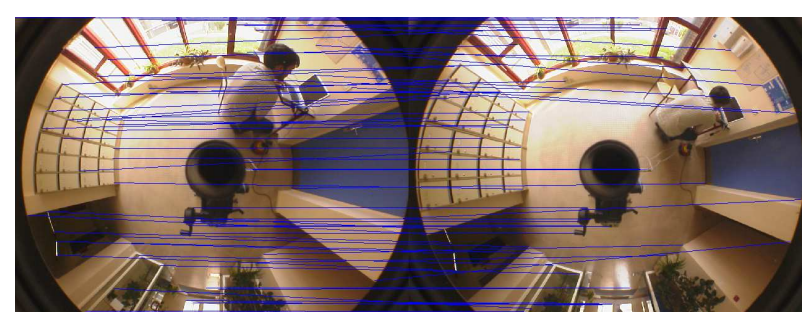

(b)

Fig. 4. Matching with ZNCC. (a) classical approach : 65 matchings, 53 true matchings,(b) geodesic approach : 71 matchings, 63 true matchings

image distorsion. We have then considered ZNCC measure :

$Z N C C(x, y)=\frac{\sum_{i \in \mathcal{V}(x)} \sum_{j \in \mathcal{V}(y)}\left(I_{1}(i)-\overline{I_{1}(x)}\right)\left(I_{2}(j)-\overline{I_{2}(y)}\right)}{\sqrt{\sum_{i \in \mathcal{V}(x)}\left(I_{1}(i)-\overline{I_{1}(x)}\right)^{2} \sum_{j \in \mathcal{V}(y)}\left(I_{2}(j)-\overline{I_{2}(y)}\right)^{2}}}$

where $\overline{I_{1}(x)}\left(\operatorname{resp} \overline{I_{2}(y)}\right)$ is the mean of $I_{1}$ (resp. $I_{2}$ ) in the neighborhood $\mathcal{V}(x)$ (resp. $\mathcal{V}(y)$ ). We have compared a classical neighborhood of size $7 \times 7$ with a geodesic neighborhood $\mathcal{V}_{r}^{3}$ (fig 4). If we consider the same corners in both cases and the same thresholds, classical ZNCC allows to match 65 points. Among these 65 matchings, only 53 are correct, which represents $18.4 \%$ of outliers. In the case of geodesic neighborhood defined by (2), we obtain 71 matchings including 63 correct ones, which is equal to $11.2 \%$ of outliers.

\section{CONCLUSION}

In this paper, we have proposed a new approach for omnidirectional image processing which consists in considering the spherical space and the geodesic metric rather than Euclidean plane with Euclidean metric. We are then able to define a geodesic neighborhood which presents several advantages :
- Masks are similar to the masks in $\mathbb{R}^{2}$

- Classical methods are easily transposable.

The single drawback of the approach deals with the necessity to perform an interpolation of the image in order to define our regular grid. Nevertheless a simple interpolation by nearest neighborhood allows to obtain good results as shown in the experiments. A very interesting perspective consists in developing spherical multi-scale image processing.

\section{REFERENCES}

[1] R. Benosman and S.B. Kang, Panoramic vision: sensors, theory, and applications, Springer-Verlag New York, Inc., 2001.

[2] I. Bogdanova, X. Bresson, J. Thiran, and P. Vandergheynst, "Scale-space analysis and active contours for omnidirectional images," IEEE Transactions on Image Processing, vol. 16, no. 7, pp. 1888-1901, 2007.

[3] J.P. Barreto, "A unifying geometric representation for central projection systems," Comput. Vis. Image Underst., vol. 103, no. 3, pp. 208-217, 2006.

[4] C. Geyer and K. Daniilidis, "Catadioptric projective geometry," IJCV, vol. 45, no. 3, pp. 223-243, December 2001.

[5] X.H. Ying and Z.Y. Hu, "Can we consider central catadioptric cameras and fisheye cameras within a unified imaging model," in ECCV04, 2004, pp. Vol I: 442-455.

[6] F. Jacquey, F. Comby, and O. Strauss, "Fuzzy edge detection for omnidirectional images," Fuzzy Sets Syst., vol. 159, no. 15, pp. 1991-2010, 2008.

[7] C. Demonceaux and P. Vasseur, "Markov random fields for catadioptric image processing," Pattern Recogn. Lett., vol. 27, no. 16, pp. 1957-1967, 2006.

[8] A. Makadia, C. Geyer, and K. Daniilidis, "Correspondence-free structure from motion," IJCV, vol. 75, no. 3, pp. 311-327, December 2007.

[9] S. Bigot, D. Kachi, and S. Durand, "Spherical edge detector: Application to omnidirectional imaging," in ACIVS, 2008, pp. 554-565.

[10] S. Ainouz, O. Morel, N. Walter, and D. Fofi, "Mirroradapted matching of catadioptric images," in IEEE International Conference on Image Processing, 2008, pp. 309-312.

[11] C. Schmid, R. Mohr, and C. Bauckhage, "Evaluation of interest point detectors," International Journal of Computer Vision, vol. 37, no. 2, pp. 151-172, 2000. 